\title{
24. Influencing Indigenous policy making with statistics
}

\section{Jon Altman and Boyd Hunter}

Providing a brief concluding chapter to a very comprehensive and data-rich volume is a challenging task. This also proved to be the case in making some concluding comments to the conference on which this volume is based. In this final chapter, we seek to combine comments made in summing up the conference with some broad-brush attempts to encapsulate key issues that have been raised, both by conference delegates and by readers when this volume was peer reviewed.

Tim Rowse raises the role of theory in scholarly analysis in his opening chapter. Without a clear theoretical framework or disciplinary perspective it is difficult to interpret statistical data. While few of the chapters in this monograph focus on theoretical issues explicitly, the majority are clearly theoretically grounded. For example, a theory of poverty is used by economists to estimate the wellbeing of households. Hence, information on levels of income is qualified by information on costs and on preferences implicit in household expenditure surveys. Hunter's chapter on income, financial stress, and social exclusion uses such theory to derive measures of equivalence scales and poverty. Clearly, underlying preferences and cost structures are not necessarily the same for all sub-population (or cultural) groups, and therefore the imposition of any particular equivalence scales can, and should, be contested. Hunter's chapter provides an example of how such contestation can be undertaken.

From an academic perspective, statistics should never just be presented atheoretically - indeed, it is a maxim of the dominant western scholarship paradigm that while sound empirical research is informed by theory, conversely, sound theory is informed by empirical research. Consequently, one cannot be divorced from the other. A complicating factor, however, is that data can be consistent with different theories either within one discipline, or informed by different disciplinary perspectives. In such circumstances, it is essential to acknowledge such contestation and the impacts that it might have on the interpretation of statistical evidence.

It is easy to make a case, especially in the emotive area of Indigenous affairs, that 'something needs to be done'. It is far more difficult to provide evidence about 'what policies will be most effective'. In addition to incorporation of theoretical models in any evaluation of the evidence, analysts need to be acutely aware of the reliability and shortcomings of any data they use. The important scene setting work by Biddle and Hunter in Chapter 3 demonstrates that one 
must be careful in how the data are used: of the size of sampling errors, the likely nature of non-sampling error, the availability of standard errors, and the misspecification of questions that do not assist in answering any policy question coherently. Given this scope for questioning the reliability of data, it is important to validate any information from a questionnaire-based social survey instrument against any existing alternate survey or administrative data sets. Aware of this need, many chapters in this monograph seek to do this. Hopefully, the discussion by Biddle and Hunter alerts those with sufficient expertise to conduct their own validation exercises if they have residual concerns about the quality of any data.

Hence, while data from the 2002 NATSISS can be used for sophisticated analysis, it is important that qualifications about data quality are clearly stipulated. The data from the 2002 NATSISS can be used for policy formulation and broad evaluation. However, such undertakings are rarely straightforward; they are generally complicated and require appropriate contextualisation, both of theoretical perspective and data quality issues. Unfortunately, popular discourse, especially in the print and electronic media, often misuses information when analysts look for a sensationalistic angle in their story. Or, alternatively, statistics are amendable to selective use to support a predetermined position. The chapter on substance use by Brady and Chikritz is illustrative of how dangerous it can be to use data of doubtful integrity without appropriate heavy qualification.

What is the quality of current information about Indigenous wellbeing? The previous CAEPR monographs that focused on national surveys for Indigenous Australians were provided to conference participants to ensure that the discussion did not become too focused on the latest survey instruments and outputs, without placing them in a historical context (Altman 1992; Altman \& Taylor 1996b). However, this is difficult, for it is clearly tempting (unless undertaking comparative analysis) to focus on what is the most contemporary.

An important issue that was raised in previous workshops in 1992 and 1996 (and resulting monographs) is the need for Indigenous identifiers in other ABS household surveys like the Labour Force Survey (LFS) and the Household Income and Expenditure Surveys. This issue did not garner much attention at the 2005 conference, but we believe it remains an important issue to consider and debate. We recognise the statistical sampling problems created by the relatively small size of the Indigenous population and the fundamentally different geographic distribution to that of other Australians. Nevertheless, there is still a case for regular augmentation in sampling frames to allow robust identification of Indigenous Australians from these mainstream surveys. The ABS has recognised this need in the annual augmentation of the LFS, and the recent release of experimental labour force statistics for the period 2002-04. These statistics are accompanied by suitable qualifications (and standard error tables) about the sensitivity of the data owing to sampling error and the need to be cautious when 
using these experimental data for comparative analysis over both time and space (ABS 2006). As the ABS is all too aware, it is extremely difficult to convey the need for such caution to the mainstream media. There are other situations where data have not been released by the ABS to date, apparently because of quality concerns. On one hand, it is undeniable that the ABS has statistical expertise to make such judgments about data quality. On the other hand, it might be preferable if such judgments were scrutinised via the peer review process rather than by in-house processes within the ABS (see discussion in Hunter \& Taylor 2001).

An associated issue is what policy questions are being addressed with the collection of social statistics. This is important because social policy questions need to be cast as a priori and theoretically-informed social science hypotheses. Typically, this concern has arisen ex-post-facto, after surveys have been completed (see chapters in Altman \& Taylor 1996b). Sometimes this has resulted from decisions on the questions to be asked being based on a process involving consultation with the diverse stakeholders represented on the survey reference group. Clearly, in the development of the 2008 NATSISS, there is room for recognising that there is a clear difference between subject expertise (both theoretical and empirical expertise) and statistical expertise. The ABS has a great deal of the latter, but probably less of the former. Social science expertise, including that of Indigenous practitioners of the social sciences, is needed to decide on the questions to be asked. It might be more productive to resolve such first order issues before taking them for approval to a survey reference group. And it might also be productive for the ABS to enhance the input of social sciences expertise at the outset and not just as one set of stakeholders on reference groups.

The issues of evaluation and the need for data for community and regional development purposes under the new arrangements in Indigenous affairs arose on many occasions during the conference. In order to conduct policy and program evaluations, there is a need for access to administrative data that appears to be increasingly shrouded in confidentiality and hence inaccessible for independent assessments. Several contributors to this volume emphasised the need for better access to administrative data sets, especially in relation to labour market programs, with specific examples raised in discussions focusing on the very limited access to Centrelink information on job-seekers and Department of Employment and Workplace Relations information on CDEP scheme participation.

The reduced availability of NATSISS data at the regional level is of potential concern, since such data could be useful for evaluating the efficacy of new administrative arrangements, especially as these emphasise local processes. While the geographic information in the 2002 NATSISS is - in some ways - more satisfactory than in the 1994 survey, this information was provided by remoteness 
classification and State, rather than the smaller regional aggregations reported earlier (e.g. ATSIC regions). Data will be needed at regional and community levels to match the jurisdictions of proposed Regional Partnership Agreements and the scores of SRAs that have now been completed. However, it is far from clear whether a survey instrument is the best means of acquiring the necessary data for these purposes, given that the SRAs are so community-focused. And yet there is a growing and worrying mismatch between the level at which data are available and the level at which they are increasingly needed. It is imperative that data are not just produced at broad remote/non-remote geographical level. Such broad levels of aggregation are unlikely to reflect the lived reality of Indigenous people and they are disconnected from the focus in the new arrangements on the community and regional level. On one hand, an instrument like the census does provide information at the community level, but it is only conducted every five years and lacks Indigenous-specific questions. On the other hand, there is a new emerging need for task-specific data collections, including qualitative data about processes, if the new arrangements in Indigenous affairs are ever to be rigorously evaluated. It is not clear to us what analogous processes to academic peer review exist in the policy community to put in place the transparency and accountability referred to in the COAG (2004) principles covering delivery of services to Indigenous people across Australia.

The 2002 NATSISS does provide some advances on previous data collection, but it still has limitations. The lack of an adequate disaggregated geography has just been noted. But to allow a higher degree of disaggregation, the NATSISS sample may need to be augmented. However, as the ABS made clear at the conference, there are obvious costs and benefits associated with such an option that need to be recognised. And while the contributions to this monograph contain many specific suggestions for improving particular questions in the 2008 NATSISS, these clearly need to be balanced against the needs for consistency in inter-temporal analysis, that is, between the 1994 NATSIS and the 2002 NATSISS.

It is important not to adopt terms like 'evidence-based policy formulation' uncritically. Almost by definition, any such policy development tends to place more emphasis on outcomes than process. Several chapters in this volume have noted an increasing emphasis on outcomes at the expense of process in recent policy initiatives. This development is not universally endorsed by the authors here. The artificial distinction between these two aspects of policy has been highlighted in debate about the relative merits of practical reconciliation (which focuses on outcomes) and symbolic reconciliation (which focuses on processes or rights). Altman and Hunter (2003) argue this distinction is unclear, unhelpful, and may militate against effective policy formation, especially if it alienates Indigenous people. Any socially-oriented policy is unlikely to work unless it secures the firm cooperation of the people whose welfare it seeks to enhance. 
One instrumental concern with outcomes-based policy is that where outcomes are poorly defined, we may get a distorted set of policies based on hitting targets rather than actually delivering benefits. An example is the CDEP scheme and its role in delivering employment equality to Indigenous Australians. Assuming employment is the desired policy outcome, the CDEP scheme can be regarded as either a huge success or a huge failure depending on whether or not people in the CDEP scheme are defined as employed. The real issue is whether CDEP participation delivers the kinds of benefits normally associated with (market-based) employment. Alternatively, if the CDEP scheme is not just about employment, then all the other social, cultural and locational benefits associated with it need to be clearly articulated and evaluated.

An evidence-based policy approach in Indigenous affairs requires transparent critical scrutiny of the analyses of both researchers and policy makers. Peer review is one way to ensure that the data presented provides, in some sense, evidence for a particular proposition. While the focus of this monograph has been on data quality and the information content in the 2002 NATSISS, it is also important that analysts and policy-makers be held accountable. This monograph is a modest contribution to this process. As academics and policy commentators, we need to be constantly on the lookout to challenge loose research (and associated loose language and loose claims of success or failure), especially if it allows gratuitous appeals to populist or emotive sentiments.

One of the potential strengths of an edited volume is that it does not impose any one view on individual contributors. While this monograph is in many ways inter-disciplinary, the peer review process encourages, and indeed requires, robust criticism of the content of various chapters. The conference provided the first opportunity for peer review, while subsequent independent academic review provided a second layer of quality control.

In the spirit of providing a critical reflection on the inter-play between theory and empirical evidence, it is of interest to focus on two chapters as exemplars of this issue, but from opposite ends of the spectrum. Will Sanders is a political scientist who is one of Australia's foremost experts on Aboriginal housing. In his analysis of housing need, Sanders uses a particular approach that focuses on populations and households and that also focuses on institutional arrangements (see also Sanders 2005). Sanders's chapter here is interesting because he takes an unorthodox position arguing that from his perspective, and for his research purposes, better data already exist in the five-yearly census than in the Indigenous-specific NATSISS. This is impossible to argue with; the proof is in the many important and sensible contributions that Sanders has made to debates about Indigenous housing over the years (see Sanders 1990, 1993, 2005). And yet it could be argued that Sanders overlooks a potential major benefit of the NATSISS: it provides information that would allow multi-factor explanations 
of Indigenous housing shortfalls beyond the institutional. We highlight the housing issue because of obvious relationships at the level of the individual between housing tenure and housing adequacy and a wide range of socioeconomic variables (including social factors, such as education, labour force status and health status). These are important areas for researchers and policy makers to examine using NATSISS rather than the census which provides an inadequate number of variables. Using NATSISS it would be possible to go beyond reliance on population estimates from the census as the basis for Indigenous housing analysis, but Sanders makes it clear that multi-variate analysis is not his particular preferred approach - he leaves that task for others, perhaps from different disciplinary perspectives.

The lack of multi-variate analysis in the housing chapter is shared by many other chapters in this monograph and is partly explained by the lack of space available to authors both at the conference and in this volume to develop a proper theoretical and empirical framework (that would differ substantially between the various subject areas). ${ }^{1}$ Survey data that include a sufficiently large number of Indigenous people can allow researchers to explore subtle behavioural relationships when informed by suitable theoretical models and appropriate multi-variate techniques. The data available from the 2002 NATSISS presents an important opportunity to conduct new analyses to inform policy makers and test theoretical propositions/hypotheses.

Bob Gregory's chapter, conversely, illustrates why one should be wary about making too many claims for theoretical propositions. Gregory uses a conventional neoclassical economics framework to argue that economic policy must be informed by an understanding of supply and demand side issues. In reality, though, it is often difficult to separately identify whether supply or demand or what mix of supply and demand are generating the outcomes - in Gregory's particular case, in the labour market. That is, while it is important to be cognizant of relevant theoretical issues, it is also vital not to make too many claims on available data. Notwithstanding this, Gregory is probably right to speculate that policies are likely to be ineffective if one ignores the distinction between supply and demand just because the available data do not allow one to separately identify the relevant issues for Indigenous Australians. While Gregory is more concerned with 'asking the right questions' than providing definitive evidence for any one proposition, this is not entirely the case in public debate where he seems to privilege supply of Indigenous labour over demand for labour and to postulate policy positions that highlight the need for labour migration when employment outcomes are not guaranteed (Gregory 2005).

1 Even if the multi-variate analysis is not always conducted in this volume, several authors allude to the importance of controlling for confounding the bi-variate analysis in their respective chapters in this volume (e.g. Gray \& Chapman; Radoll). 
One of the continual tensions for the ABS is the extent to which it should include and frame questions in future NATSISS data collections that will either allow direct comparisons with non-Indigenous Australians or include questions that have the greatest relevance for Indigenous people themselves. To the extent that one leans towards the latter, it will be necessary to frame questions in the most culturally appropriate way - a process which ideally should be determined, in terms of appropriate phrasing of the question, with extensive input from Indigenous people themselves. However, the diversity in cultural practice across Indigenous Australia means that even the most sensitive data collection techniques will be unreliable to a certain extent (i.e. have a certain level of non-sampling error). The inherent unreliability of survey data on cultural variables was identified in Nic Peterson's chapter, especially the difficulty in interpreting the changes in culture and identity between 1994 and 2002. While cultural data are exceptionally sensitive to the methodology and general context of a survey, one should not underestimate their importance as they are essential background for many of the theoretical perspectives used to interpret data for Indigenous Australians. This may be an intractable problem: despite the best intentions of the ABS, surveys may be the wrong instrument to collect cultural data and more field-based ethnographic techniques are likely to better capture diversity.

If the policy vision for the future of Indigenous Australia is 'equality' in the sense of Indigenous people achieving the same outcomes as non-Indigenous peoples, then greater emphasis should be placed on collecting data which will allow direct comparisons with non-Indigenous Australians. Whether questions are framed in a culturally sensitive way would be a secondary concern to that of achieving direct comparability with existing general population surveys. This also pre-supposes that ultimately Indigenous Australians will share the same aspirations as non-Indigenous Australians, ultimately a culturally determined presupposition.

If, on the other hand, the policy vision is cultural plurality, self-determination and choice, then comparability with general population data would be of secondary concern. Much discussion in this monograph can be seen in this light, including frequent queries being raised about different techniques and questions used in remote and non-remote areas (or CAs/NCAs). Such differences presumably arose in a survey of Indigenous Australians from a range of factors, including:

- recognition of different aspirations and degrees of integration with non-Indigenous society

- $\quad$ issues of cultural appropriateness in survey design and conduct

- treatment of CDEP jobs as employment in most contexts

- laments about the absence of adequate data on the 'customary economy', and 
- inadequate recognition of hunting, gathering and fishing activities as legitimate economic activities (see chapters by Biddle \& Hunter; Altman, Buchanan \& Biddle; and Taylor \& Kinfu).

It is noteworthy that the tension we are highlighting here has been embedded in both policy and disciplinary approaches for many years now. On the policy front, Altman and Sanders (1991) highlighted the tension between equality and equity in the now superseded Aboriginal Employment Development Policy (AEDP). The AEDP sought statistical equality between Indigenous and non-Indigenous Australians by the year 2000. The measurement of attainment of this policy goal would need comparable statistical information on Indigenous and other Australians, with the five-yearly census being the obvious instrument. However, the AEDP also sought to recognise cultural diversity, connoted by the term 'equity' (or fairness). Measuring this would not only require statistics about cultural differences, but would also require trade-offs with the goal of statistical equality. For example, if on equity grounds people chose and were supported to live at remote localities distant from mainstream opportunities, their chances of equality would be heavily circumscribed.

On the disciplinary front, Altman and Rowse (2005: 159-77) have recently highlighted a similar tension within the social sciences differentially interpreting the goals of Indigenous affairs policy as either of socioeconomic equality or the facilitation of choice and self-determination. Using anthropology and economics as exemplars, they have shown how anthropology dwells on cultural difference and presumes that difference to be a social good, whereas economics dwells on socioeconomic inequality and presumes that it is the negative legacy of historic exclusion, racism and neglect. There is clearly a tension between the two ideals of 'equality' and 'plurality' in a liberal democracy like Australia, and associated challenges to statistics that seek to measure 'equality' in some extremely remote, difficult and structurally constrained circumstances where many Indigenous people choose to live.

Perhaps the most common sentiments expressed by the authors of chapters in this monograph were hopes for enhancements to the data through greater comparability (particularly between the 2002 NATSISS and future national surveys), merging or supplementing the survey data with administrative data collections, and collection of true longitudinal or panel data. While strong arguments can be made for all of these propositions, it is important to acknowledge that all such strategies are not without cost. Matching ABS statistics with departmental records over time, or establishing a 'true' longitudinal data set (focusing on the same individuals over time), may not be an optimal use of scarce resources - both approaches are expensive and the resulting data introduce 
their own sets of limitations for researchers. ${ }^{2}$ However, tracking individuals across time can potentially offer insights into causal relationships and dynamics of social interactions, both of which are almost impossible to examine with cross-sectional data. Consequently, it is vital to consider how longitudinal data are best collected for Indigenous Australians. The experience of the Longitudinal Study of Indigenous Children, which is currently being designed by FaCS and should be in the field in 2007, will itself provide data on how future longitudinal data might be collected for Indigenous Australians.

This monograph covers a wide range of research topics, and this obviously reflects the very wide range of issues about which the 2002 NATSISS collected statistical information. There is an inevitable trade-off between the depth to which any issue is explored and the number of different issues canvassed. This can be seen as reflecting either positively or negatively on the survey. Almost invariably, writers in each subject area pointed to both positive aspects of the 2002 data and to items they felt could have been included or expanded upon for the purposes of their own research.

This publication contains numerous recommendations, and ultimately it will not be feasible for the ABS to adopt all, or even most, of them. Indeed, the main aim of this monograph is to stimulate public debate about Indigenous issues among policy makers, academics, and other users of ABS data. While, as noted at the outset, detailed data from the 2002 NATSISS only became available last year, it is important that debate about these important statistics is disseminated as widely as possible. It is unfortunately the case that the numbers that can attend a two-day conference in Canberra will always be limited. Hopefully, this publication with its diverse perspectives and subjects, mainly drawn from the 2002 NATSISS, should make a contribution to the quality of analyses of Indigenous affairs issues and the overall standard of Australian public debate. It is our hope that the contents of this monograph might improve the overall standard of public discourse on Indigenous issues by highlighting some of the statistics that are currently available, when and for what they can be used, and when we need to take extreme care in how we use them.

2 However, Gray \& Hunter illustrate that synthetic panel data can be constructed to partially redress the lack of 'true' longitudinal data for Indigenous individuals (Gray \& Hunter 2002; Hunter \& Gray 2001). 